\title{
Examining the clinical trial feasibility process and its implications for a trial site
}

\author{
This article was published in the following Dove Press journal: \\ Open Access Journal of Clinical Trials \\ 8 September 201 I \\ Number of times this article has been viewed
}

\section{LJ Burgess}

NU Sulzer

TREAD Research/Cardiology Unit, Department of Internal Medicine, Tygerberg Hospital and Stellenbosch University, Parow, South Africa
Correspondence: LJ Burgess TREAD Research/Cardiology Unit, Department of Internal Medicine, Tygerberg Hospital and Stellenbosch University, Parow 7505, South Africa

$\mathrm{Tel}+27219317825$

Fax +27 21 9333597

Email lesley@treadresearch.com
Objectives: To retrospectively analyze feasibility questionnaires to evaluate the number of trials that resulted in patient enrolment and the mean time frame involved.

Methods: This study was conducted by TREAD Research, a site-managed organization based in the Western Cape, South Africa, between January 2004 and December 2009. All feasibility questionnaires received by the site over this time period were analyzed. Descriptive statistics were used to analyze the data.

Results: A total of 252 feasibility questionnaires were received; 207 were accepted and 45 rejected. An average of $26.8 \%$ of trials started out of those feasibilities that were accepted by the site. The average time frame from feasibility acceptance to patient enrolment was 12.9 months (range 2.7-33.5 months).

Conclusion: Improving the trial feasibility process would markedly improve a trial site's ability to plan effectively and efficiently allocate appropriate resources.

Keywords: resource allocation, business planning, clinical research organizations

\section{Introduction}

Pharmaceutical companies and clinical research organizations (CROs) typically assess a clinical trial site's potential for patient recruitment through a feasibility questionnaire. Sites are sent a synopsis of the proposed trial and requested to answer various questions relating to the site's clinical trial experience, its facilities, previous experience with recruiting for a particular therapeutic area, and its ability to access the required patient population. Based on these feasibilities, sites are chosen to participate in the proposed trial.

A number of shortfalls have been noted in this process. In particular, sites are frequently supplied with a protocol synopsis that does not accurately reflect the final protocol, and the target patient population is not detailed. This makes subsequent planning on both the pharmaceutical company's and/or CRO's as well as the trial site's behalf, extremely difficult. Another criticism of the feasibility process is that it is to a large extent subjective. Typically, the selection of countries and investigators for clinical trial participation has been based on a combination of unsubstantiated feasibility questionnaires and familiarity with a core group of investigators. ${ }^{1}$ Additionally, the completion of a feasibility questionnaire does not guarantee that the trial site will be awarded the trial and, even if the trial is awarded, no set time frame is provided from the completion of the feasibility questionnaire to patient enrolment. This fact further compounds the difficulty of planning from the trial site's perspective. 


\section{Objective}

The primary objective of this study was to retrospectively analyze the feasibility questionnaires received by a trial site in order to: (1) compare those feasibilities rejected to those accepted by the site; and (2) compare feasibilities issued by pharmaceutical companies as opposed to CROs. The secondary objectives were to: (1) evaluate the number of trials that resulted in patient enrolment out of the total number of feasibilities received, and (2) assess the mean time frame from acceptance of a feasibility questionnaire to enrolling the first patient.

\section{Methods}

This retrospective analysis was conducted by TREAD Research, a site-managed organization based within an academic hospital in the Western Cape, South Africa, between January 2004 and December 2009. All feasibility questionnaires received by the site over these 6 years were analyzed, and the data were entered into a Microsoft Excel ${ }^{\circledR}$ spreadsheet (Microsoft, Redmond, WA). Data captured included year of feasibility questionnaire receipt, the company from whom the feasibility was received (pharmaceutical company or CRO), whether the feasibility was accepted or rejected by the site, the reason for rejection (if applicable), and finally those feasibilities that resulted in patient enrolment, including the time from feasibility completion to first patient enrolled. Descriptive statistics were used to analyze the data.

\section{Results}

A total of 252 feasibility questionnaires were received over the 6-year period. Table 1 presents a summary of the questionnaires per year according to the number accepted $(n=207)$ and those rejected $(n=45)$. The reasons for feasibility rejections included inappropriate therapeutic area for this trial site (44\%), competing studies (31\%), pharmaceutical company/CRO delay (13\%), and other (12\%).

The percentage of trials that resulted in patient enrolment from those feasibility questionnaires that were accepted by the site, per year, is presented in Figure 1. The average percentage of trials initiated from those feasibilities that

Table I Feasibility questionnaires accepted and rejected by the trial site per year $(n=252)$

\begin{tabular}{lllllll}
\hline Feasibility questionnaires & $\mathbf{2 0 0 4}$ & $\mathbf{2 0 0 5}$ & $\mathbf{2 0 0 6}$ & $\mathbf{2 0 0 7}$ & $\mathbf{2 0 0 8}$ & $\mathbf{2 0 0 9}$ \\
\hline Feasibilities accepted & 25 & 28 & 37 & 48 & 39 & 30 \\
Feasibilities rejected & 0 & 8 & 6 & 9 & 14 & 8 \\
Total feasibilities received & 25 & 36 & 43 & 57 & 53 & 38 \\
\hline
\end{tabular}

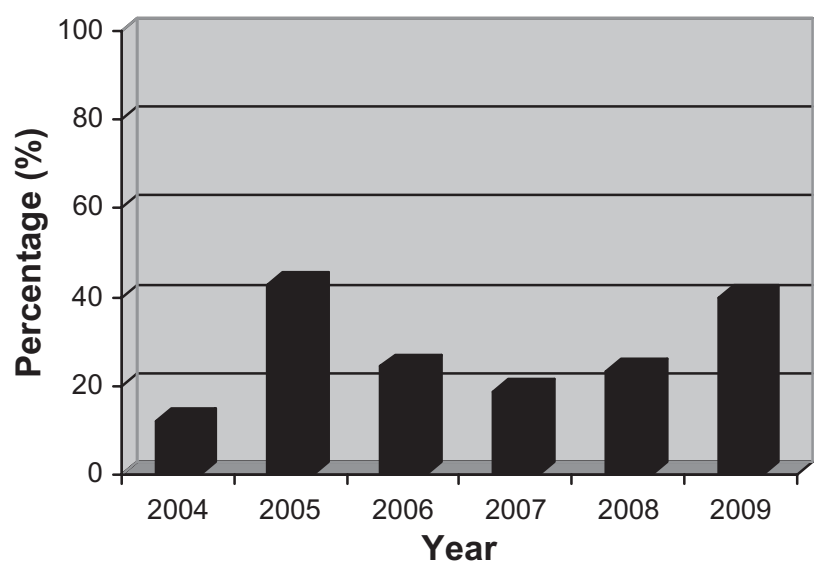

Figure I Percentage of trials started out of those feasibility questionnaires that were accepted by the site, presented per year.

were accepted by the site was $26.8 \%$. A further analysis compared the number of trials started from feasibilities accepted between pharmaceutical companies and CROs. The data is presented in Figure 2. Pharmaceutical companies consistently had more trials that resulted in patient enrolment than CROs.

The final analysis calculated the mean time from feasibility return to first patient enrolled per year, as well as overall for the 6 years combined. The findings are presented in Figure 3. The average combined time frame for the 6 years was 12.9 months, although this ranged from a minimum of 2.7 months to a maximum of 33.5 months (2.8 years) from feasibility return to first patient enrolled.

\section{Discussion}

The results of this study demonstrate that, at this site, only a small percentage $(26.8 \%)$ of feasibilities accepted by the site result in enrolling studies and that the majority of these trials are initiated by pharmaceutical companies. This study

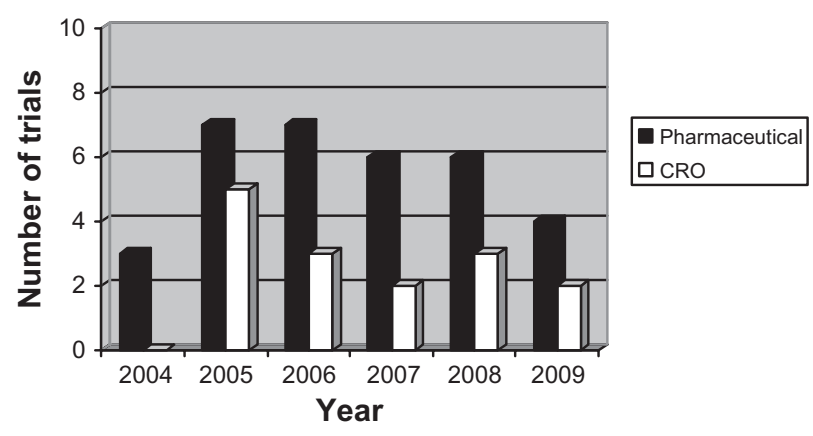

Figure 2 Number of trials that resulted in patient enrolment per year for pharmaceutical companies and CROs.

Abbreviation: CRO, clinical research organization. 


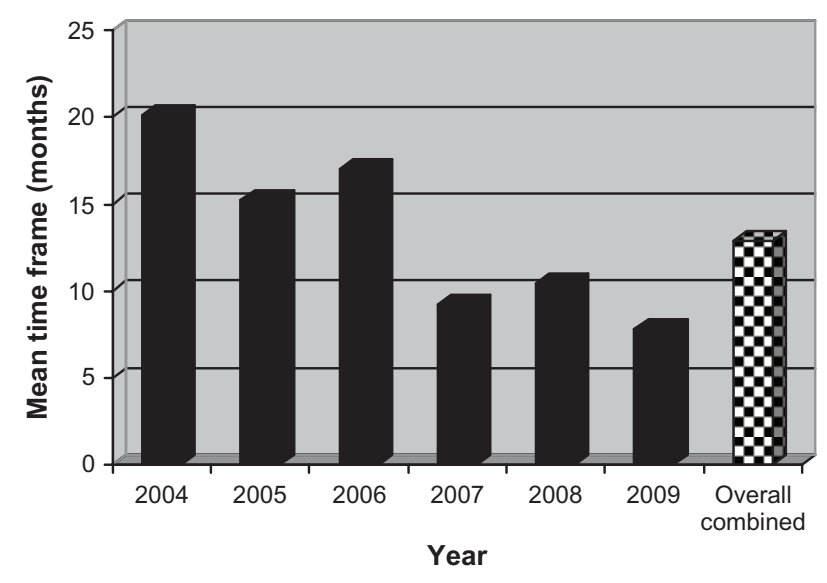

Figure 3 Average time frame (months) from feasibility questionnaire return to first patient enrolled per year and overall combined.

also demonstrated that the average time from accepting a feasibility questionnaire to patient enrolment at this site is 12.9 months (range 2.7-33.5 months).

Clinical trials are becoming more complicated, recruitment requirements are becoming increasingly stringent, and study budgets are being closely scrutinized; all of these factors result in a far more complex environment for trial sites than ever before. ${ }^{2,3}$ Clinical trial sites are arguably first and foremost businesses, yet the practical application of financial principles and business processes continues to be a challenge for many trial sites.

The small number of feasibilities accepted by the trial site that result in enrolling studies makes it extremely difficult for sites to implement effective recruitment strategies, and invariably huge administrative and human resources are wasted. Furthermore, the trial site has little, if any, control over the decision processes that determine which studies result in enrolment. Some of the factors which may affect whether a study at feasibility level actually enrolls patients include the pharmaceutical company's budget changes, drug or trial timeline delays, a novel agent's unexpected and unfavorable clinical performance, alterations to a pharmaceutical company's strategic planning, delays in regulatory approval processes, and in some instances, failure on the CRO's part to secure a trial. None of these factors are under the control and/or influence of the trial site in any way, yet these decisions play an integral role in the site's recruitment potential and therefore ultimate financial survival.

A further finding of relevance is the average time taken from the acceptance of a study's feasibility to the enrolment of the first patient. The average time for the 6 years combined was 12.9 months, although this ranged from a minimum of
2.7 months to a maximum of 33.5 months ( 2.8 years). This delay in getting studies initiated, combined with the huge range in time periods from study feasibility to patient enrolment, renders strategic business planning, budgeting, and resource allocation almost impossible. This unpredictable situation also places enormous stress when setting up new trial sites, as determining time lines, cash flow, and budgets can almost never be accurate as the factors involved are too variable.

Arguably, one of the main local determinants in the lengthy process from feasibility acceptance to patient enrolment has been the regulatory approval process. The Medicines Control Council has been criticized for its inefficiency and administrative delays in approving clinical trial applications. Recent developments suggest, however, that these matters are being addressed and approval timelines are improving. The data presented in Figure 3 seems to support this and suggests a trend towards reduced timelines from 2007. This is of paramount importance in attracting foreign pharmaceutical companies to conduct research in South Africa.

South Africa currently enjoys approximately $0.6 \%$ of the world clinical trial market share, with the capacity to increase to $2.5 \%$. The South African Clinical Research Association estimates that in 2008, approximately R2.2 billion was generated through conducting internationally sponsored randomized controlled trials in South Africa. ${ }^{4}$ Competition from other emerging markets such as South America, China, and emerging Eastern European countries is growing, and there needs to be a concerted effort as a country to address any issues that may discourage further investment in the local clinical trial industry.

\section{Conclusion}

The clinical research process is time and human resource intensive, ${ }^{3}$ and the allocation of resources, along with strategic planning and budgeting, are daily challenges. The feasibility process is a complicated and arguably flawed one, influenced by numerous factors, some of which are not intrinsic to the trial site, yet invariably play a direct role in determining a trial site's success or failure. Improving the trial feasibility process and minimizing the range in time lines between feasibility acceptance and patient enrolment would markedly improve a trial site's ability to plan effectively and efficiently allocate appropriate resources.

\section{Disclosure}

The authors report no conflicts of interest in this work. 


\section{References}

1. Jones M, Jones S. Data based predictions. Applied Clinical Trials. March 1, 2008. http://appliedclinicaltrialsonline.findpharma.com/ appliedclinicaltrials/article/articleDetail.jsp?id=500434. Accessed April 28, 2010.

2. Drayton S, Fraser HE. Five steps to faster recruitment. Good Clin Pract J. 2005 Feb:17-20.
3. Snyder A. CTMS can provide business intelligence for sites - Applied Clinical Trials. 2010 July 1. http://appliedclinicaltrialsonline.findpharma.com/ appliedclinicaltrials/Articles/CTMS-Can-Provide-Business-Intelligencefor-Sites/ArticleStandard/Article/detail/678140. Accessed July 26, 2010.

4. Richardson M-A. Focus on Africa. 2009 Nov. Available from: http:// www.acro.co.za. Accessed December 2009.

\section{Publish your work in this journal}

The Open Access Journal of Clinical Trials is an international, peerreviewed, open access journal publishing original research, reports, editorials, reviews and commentaries on all aspects of clinical trial design, management, legal, ethical and regulatory issues, case record form design, data collection, quality assurance and data auditing methodologies. The manuscript management system is completely online and includes a very quick and fair peer-review system, which is all easy to use. Visit http://www.dovepress.com/testimonials.php to read real quotes from published authors.

Submit your manuscript here: http://www.dovepress.com/open-access-journal-of-clinical-trials-journal 